\title{
Certificación del médico general
}

José Adrián Rojas-Dosal

Presidente del Comité Normativo Nacional de Medicina General (CONAMEGE), México

La regulación de la profesión médica es un proceso antiguo en nuestro país. Desde los tiempos del virreinato existía el Real Tribunal del Protomedicato, que tenía como función examinar a los médicos, cirujanos y boticarios para ser autorizados a ejercer su profesión; con el tiempo, esta función se fue relegando en otras estructuras administrativas que se fueron modificando, pero que la mantuvieron en vigor.

Más recientemente, en la década de 1970, la Academia Nacional de Medicina encabezó todo un proceso encaminado a la certificación de los médicos especialistas, que tuvo un éxito rotundo y que actualmente permite la certificación de los médicos especialistas a través de sus Consejos.

Dado el éxito alcanzado en este rubro, en el año de 1997, el Dr. Octavio Rivero Serrano, que en ese momento era el secretario del Consejo de Salubridad General, consideró conveniente diseñar un procedimiento semejante para llevar a cabo un proceso de certificación de los médicos generales, con el objetivo de lograr una mejora en la calidad y en la seguridad de la atención de los pacientes, por medio de la preparación y la actualización de este importante grupo de profesionistas, ya que no contaban con un proceso equivalente.

Convocó a la Academia Nacional de Medicina y a la Academia Mexicana de Cirugía para que se diseñaran y organizaran el proceso de certificación de los médicos generales, dada la experiencia que estas instituciones habían adquirido con la certificación de los médicos especialistas, y de esta manera asegurar a la sociedad mexicana que también los médicos generales contaban con los requerimientos necesarios para prestar una atención médica de calidad.

Se logró establecer los acuerdos necesarios y el compromiso con todas las instancias que participarían en la certificación. Se consideró importante la organización de consejos estatales en cada una de las entidades federativas, que dependerían del Consejo Nacional, para de esta manera facilitar la ubicación de los médicos practicantes en cada una de ellas.

Finalmente quedó establecido el Comité Normativo Nacional de Medicina General (CONAMEGE), integrado por representantes de la Academia Nacional de Medicina, la Academia Mexicana de Cirugía, el Consejo Nacional de Certificación en Medicina General y como asesor externo la Asociación Mexicana de Facultades y Escuelas de Medicina; esta institución entró en funciones el año 2001.

El objetivo primordial de este organismo es la certificación de los médicos generales de todo el país por medio de la evaluación de sus conocimientos y sus competencias, además de impulsar el mantenimiento de la vigencia de esta certificación en periodos de 5 años, que se logra al acreditar una formación médica continua.

El proceso de certificación es voluntario para los médicos generales; implica valores, ética y profesionalismo por parte de ellos, al concientizar de que es indispensable asegurar a sus enfermos que están capacitados para ofrecerles una atención de calidad, dado que la certificación demuestra que están actualizados en sus conocimientos y habilidades.

En lo que concierne a la recertificación, es indudable que la rápida evolución del conocimiento y de la tecnología hace que, en un tiempo corto, quede obsoleto lo que se tenía por verdadero, y de ahí la necesidad de mantener una actualización de los conocimientos que pueda ser demostrable.

Un punto importante que también desempeña CONAMEGE es la evaluación y la puntuación de los cursos que se ofertan a los médicos generales por medio del Subcomité de Educación Médica Continua.
Fecha de recepción: 04-10-2017

Fecha de aceptación: 05-10-2017

DOI:10.24875/GMM.17003809
Gac Med Mex. 2017;153:651-652

Contents available at PubMed www.gacetamedicademexico.com 
Debe ser considerado que no todos los cursos que se ponen a disposición de los médicos generales están enfocados a sus necesidades o son de la calidad necesaria, al tener algunos de ellos sesgos con fines de publicidad o promoción de productos farmacéuticos, por lo que se da una mayor valoración con mejor puntuación a los cursos que están dirigidos a la atención primaria, con temas de prevención y manejo de los padecimientos más comunes en la población mexicana; además de tomar en cuenta las instituciones educativas que los avalan, la organización de todo el proceso educativo y la participación de profesores destacados.

Como ejemplos de oportunidades de actualización, la Academia Nacional de Medicina ha puesto a disposición de todos los médicos el curso recientemente actualizado de PRONADAMEG, que puede ser tomado en línea. La Academia Mexicana de Cirugía mantiene un diplomado de actualización para el médico general en sus instalaciones y por vía electrónica, y hay otras instituciones de salud y educativas, así como sociedades médicas, que de manera permanente ofrecen cursos de actualización con temática diversa.

Actualmente se tiene el registro de 30,095 médicos generales certificados, que mantienen su certificación vigente. Aunque no se tiene una cifra fidedigna del número de médicos generales que realizan su actividad profesional en nuestro país, es un número considerable que se acerca cuando menos al $30 \%$ de todos ellos.

Los motivos por los que el médico general se debe certificar se sustentan en varias razones. La primera es demostrar que cuenta con los conocimientos y habilidades para brindar una atención de calidad a los enfermos que ponen en sus manos, con confianza, su salud y en ocasiones su vida. Debe demostrar, también, que se mantiene actualizado durante toda su vida profesional, dado que la rápida evolución de los conocimientos y de la tecnología obligan a mantener vigentes sus conocimientos y capacidades.

También la certificación es un mecanismo que permite hacer comparables las competencias que debe tener un médico al concluir sus estudios. Actualmente hay identificadas y en funcionamiento 162 escuelas de medicina, distribuidas en todo el territorio nacional, de las cuales están egresando cerca de 20,000 médicos al año. No todas estas escuelas cuentan con la acreditación necesaria y, por lo tanto, no se conocen los programas curriculares que imparten, lo que da lugar a una gran disparidad de los conocimientos y de la calidad de los egresados de estas instituciones formativas. Un examen de certificación puede paliar algunas deficiencias en este sentido.

Una razón más es que con alguna frecuencia, en el mercado laboral, instituciones de salud, oficiales o privadas, así como instituciones educativas, exigen la certificación para su contratación. Un ejemplo es la Facultad de Medicina de la Universidad Nacional Autónoma de México, en la que se tiene considerado, en los Criterios para la Evaluación del Personal Académico de la Carrera de Médico Cirujano, que para la contratación de profesores de ciclos clínicos hasta el quinto semestre puede estar a cargo de esta enseñanza un médico general certificado.

Por otra parte, recientemente se ha establecido por el Consejo de Salubridad General un premio al mérito médico dirigido a los médicos generales, denominado Doctor Miguel Francisco Jiménez, para quienes se hayan destacado en su labor en la atención primaria de la salud y que sería deseable fuera otorgado a un médico certificado.

Finalmente, la Academia Nacional de Medicina, dando ejemplo de congruencia, tiene sillones disponibles para los médicos generales que cumplan con los requisitos para ocuparlos, entre otros el de tener su certificación vigente. 